\title{
A Discussion on the Two Aspects of natura non facit saltus
}

\author{
Chetan Datta Poduri \\ Department of Biotechnology, Sreenidhi Institute of Science and Technology, India
}

Copyright $(2016$ byauthors,allrightsreserved.Authorsagreethatthisarticleremainspermanentlyopenaccessunderthetermsofthe CreativeCommonsAttributionLicense4.0InternationalLicense

\begin{abstract}
This article discusses the popular Aristotelian phrase "natura non facitsaltus" using two possible approaches - a gradual evolution and a punctuated evolution. In doing so this communication shows that in whatever aspect we examine Nature, at a microscopic level we find that continuity exists. Perhaps the same occurs at macromolecular/multi-cellular levels. Nevertheless, in sexually reproducing organisms or in organisms which exhibit recombination, some amount of jumping occurs which cannot be considered random. Despite this, on a broader scale, nature seems to evolve continuously and gradually.
\end{abstract}

Keywords Evolution, Jumps, Continuity, Nature, HCV, Influenza Virus

\section{Introduction}

The phrase natura non facitsaltus (translated as nature does not jump) has been the crux of intense debate in the scientific community for over two centuries. Indeed the opening chapters of any popular or standard microbiology texts include a treatise on this topic. Natura non facitsaltus is traditionally interpreted as continuity between living and non-living matter, and thence a spontaneous evolution of life. Scientists of yore, viz., Redi, LazzaroSpallanzani, Louis Pasteur, seemed to have systematically disproved this by their well conceived experiments leading to statements such as 'a gap exists between life and non-living matter' [1]. The most popular classic experiment by scientists of yore in this direction included demonstration of preventing air or flies from entering glass jars whencemaggots do not grow in rotting meat placed inside the jars. Nevertheless, presented here are some arguments suggesting an absence of a jump in the Nature. In this process, the statement natura non facitsaltus is interpreted in two ways. Firstly, the continuity of life from non-living aspects like atoms is explored. This aspect is dealt as 'Continuity of Nature'. And in the second the very process of evolution itself is examined wherein some gaps that are formed naturally, referred to as 'Punctuated Gradualism', are explored with examples of bacteria and viruses. Consequently, extrapolating these arguments to larger organisms, although fossil records may show a jump in the evolutionary processes, it is indeed transient, gradual and not random.

\section{Materials and Methods}

Access to the references cited herein this article is through author's personal subscription to Academic OneFile Infotrac database (provided through the British Council Library, Hyderabad, India).

\section{Discussion}

\subsection{The Continuity of Nature}

\subsubsection{At an atomic level}

Sub-atomic particles, viz., electrons, protons, neutrons, are universal in the sense that these are the constituents of atoms whether they are present in inanimate matter or living beings. The same can be said of atoms. Continuing the discussion in similar lines, atoms constitute small molecules, viz., amino acids, nucleotides, which have been demonstrated to be present in both non-living (meteorites) and animate matter. Small molecules make up larger molecules like proteins and nucleic acids. These larger molecules are found extensively in prions, viroids, virusoids and viruses. These extremely lower level organisms may have survived in the primordial Earth in some 'cellular soup' like material. This 'cellular soup', in turn, would have comprised of molecules and atoms. Over time, this 'cellular soup' may have formed into some primitive prokaryotic and eukaryotic cells. This subsequently led to higher levels of organisms. All of these processes took place over millions of years. Thus, in this argument, natura non facit saltus appears true as continuity exits.

Nevertheless, the readers must note at this stage that viruses represent one major link between living and non-living organisms For example viruses are obligate intracellular parasites whence within the host cells they exhibit the characteristics of living organisms. Outside the host cell, although they retain their capability to infect potential host cells, they do not exhibit any activity that is 
hallmark of living organisms. Also, in certain cases some of the viruses are crystallisable. But this argument represents a classical paradox in biological sciences - which came first thechick or the egg? Similarly, because viruses require a living cell for survival, the question that crops up here is which came first - the cellular soup or the virus? Probably both evolved/formed simultaneously or a parallel evolution might be in vogue. However, to answer these questions, our knowledge of the Nature and its processes might be considered as still in its infancy and hence any attempt to provide an answer to these paradoxes might appear as amateurish.

\subsubsection{Evolution, in Bacteria, is also continuous}

Readers must keep in mind, at this stage of the arguments, that evolution is an inherent property of molecules like proteins and nucleic acids. Therefore, once cellular entities have formed, they keep undergoing evolution as it is an inherent property of the constituent molecules. This evolution continues not only at sub-cellular level, but also at a cellular level. This is best exemplified by the experiments of Elena et al [2] who have demonstrated evolution in Escherichia coli over 10,000 generations. However, the readers must note that in these experiments Escherichia coli remained Escherichia coli in the sense that the authors do not document any strain or species change, and perhaps a genera change is too much to expect in a small scale experiment wherein only two parameters were monitored. As we shall see subsequently, when we look into a similar situation in viruses, a drastic change is observed leading to complications in treating patients.

Continuous evolution on a broader perspective leads to emergence of new species, and thus newer organisms. Such an evolution has been argued to be either punctuated or gradual. Whereas punctuated refers to short rapid bursts, slow changes over a period of time is the hallmark of gradualism. Punctuated equilibrium was originally believed to be the prerogative of sexually reproducing organisms. Again, experiments of Elena et al [2] suggest that punctuated equilibrium can occur in non-sexually reproducing organisms as well [3].

\subsection{Punctuated Gradualism}

\subsubsection{In Influenza viruses}

Malmgren et al [4] argue that evolution is "punctuated gradualism". This means that in living world evolution which is slow (gradual), unfolding takes a sudden leap (punctuated) as a consequence of perhaps sexual reproduction or similar phenomenon like recombination. A classic example of punctuated gradualism in nature is the phenomenon of antigenic drift and shift observed in the evolution of Influenza viruses. Influenza viruses are RNA viruses with segmented genome. Recombination, among the viral genetic elements, in Influenza viruses is documented to be a frequent occurrence leading to evolution of newer strains. Antigenic drift is a slow change in the protein sequences of the virus leading to a decreased recognition by the immune system and can be compared to gradualism. On the contrary, antigenic shift, comparable to punctuated equilibrium, is a sudden and dramatic change in the antigenicity of the virus owing to recombination of the segments in the virus genome [5]. Thus, influenza virus exhibits 'punctuated gradualism' leading to the formation of novel strains. Malmgren et al [4] suggest that punctuated gradualismmay not be random.

\subsubsection{Other viruses not far away from Punctuated gradualism}

We can extrapolate the above arguments to other RNA viruses like hepatitis $\mathrm{C}$ virus (HCV) and human immunodeficiency viruses (HIV). The scientific community has clearly documented these viruses to exist as quasispecies and genotypes. Whereas quasispecies typically refer to the pool of viral genomes occurring within or isolated from a given individual, the different genomes of the virus isolated from various individuals are often termed as genotypes. Various studies have clearly indicated that changes in viral quasispecies dynamics play an important role in predicting the outcome of therapy imparted, at least in HCV infection [6,7]. However, it must be noted that viral dynamics in therapy represents an artificial scenario and perhaps what is true in such scenarios for small organisms may also be true in natural settings as there will be immune pressure on the viruses. Natural recombinants of HCV have been documented $[8,9]$. Nevertheless, more information is awaited before any conclusive statement regarding 'punctuated gradualism' of such recombinants can be made.

Therefore, although apparently evolution appears punctuated, because it is gradual, on the timescale it eventually manifests as a continuous process. Note that here the terms gradual and continuous have been equated. In other words Nature demonstrates continuity in evolution. Thus, natura non facit saltus.

\section{In Conclusion}

The implications of the arguments presented here include relooking the classical experiments and what the scientists of yore actually meant. Perhaps the earlier researchers were aggressive in their interpretation of natura non facit saltus. They expected instantaneous results from their experiments. This is understandable taking into account the influence of the religious authorities in their times, which at best can be described as non conducive and at worst cruel. Given the right conditions and appropriate amount of time (read as millions of years) there will be 'spontaneous' generation of life from inanimate matter as has been in the past. Thus, whatever aspect of Nature we consider, there is continuity. Also, in higher multi-cellular organisms absence of fossils that serve as connecting link to the established scheme of evolution does not mean that Nature jumps in evolution. Put simply, natura non facit saltus. All of this is summarized as figure 1. 


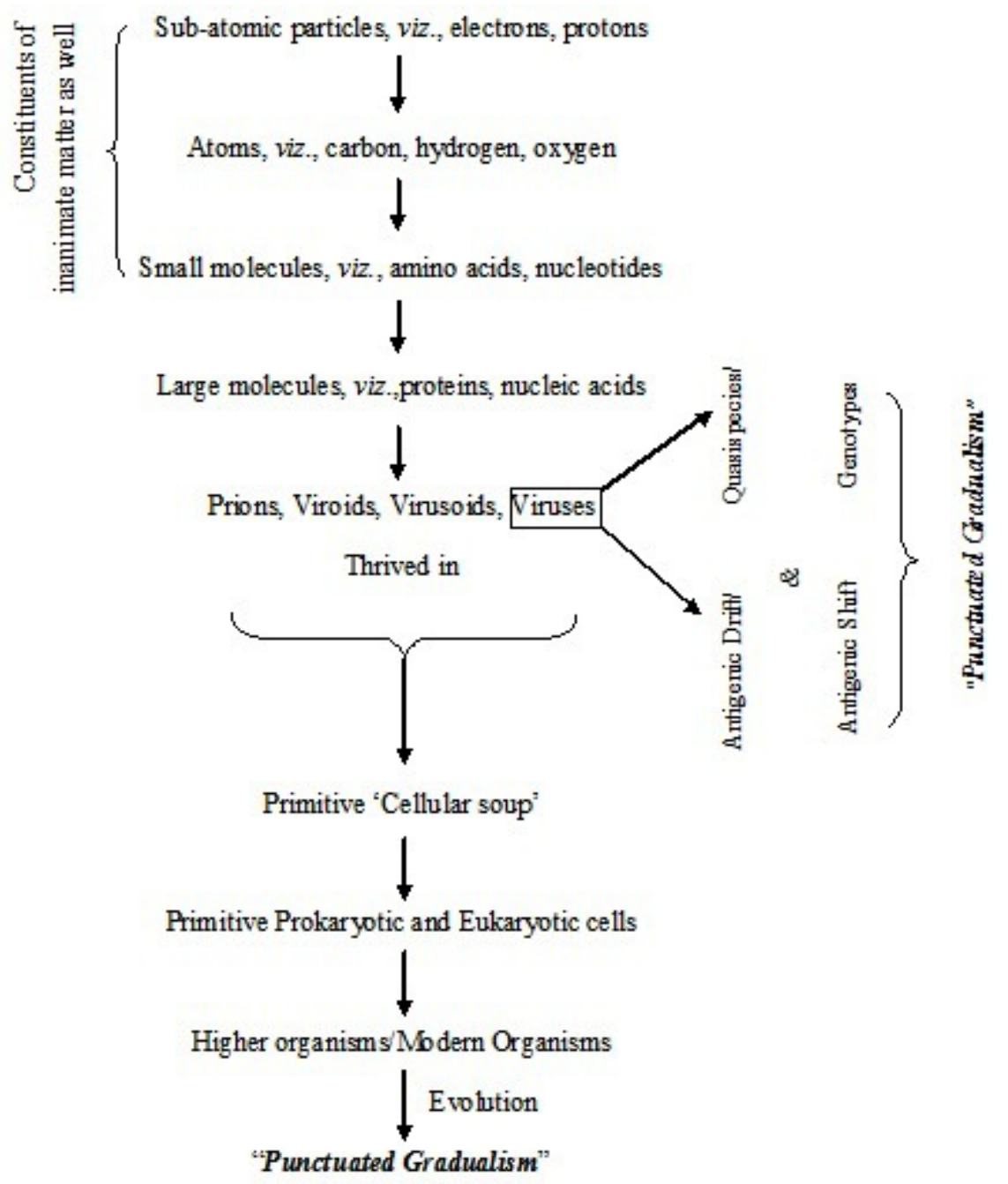

(Note the absence of jumps in this flow chart)

Figure 1. The Continuity of Nature

Overall, the arguments presented here also suggest relooking the evolution of micro-organisms like viruses with a different perspective of a 'punctuated gradualism'. Such an effort will open up new vistas in the area of virology, in particular, and the field of Evolution, in general.

\section{Acknowledgements}

The author wishes to acknowledge helpful discussion with colleagues past and present. Comments of the anonymous reviewers' which helped improve the clarity and quality of the discussions presented in this article are gratefully acknowledged.

\section{REFERENCES}

[1] Mazzarello P. A Unifying concept: the history of cell theory. Nature Cell Biol, 1999, 1, pp. E13 - E15.

[2] Elena, S. F., Cooper, V. S., and Lenski, R.E. Punctuated evolution caused by selection of rare beneficial mutations. Science, 1996, 272.5269, pp. 1802 - 1804.

[3] Mlot C. Microbes hint at a mechanism behind punctuated equilibrium. Science, 1996, 272.5269, pp. 1741.

[4] Malmgren BA, Berggren WA, Lohmann GP. Species formation through punctuated gradualism in planktonic foraminifera. Science, 1984, 225, pp. $317-319$.

[5] Cann, A. J. Principles of Molecular Virology 3rd Edition London, UK, Elsevier Academic Press, 2001, pp 192 - 195.

[6] Neumann, A. U., Lam, N. P., Dahari, H., Gretch, D. R., Wiley, T. E., Layden, T. J., Perelson, A. S. Hepatitis C viral dynamics in vivo and the antiviral efficacy of interferon-alpha therapy. Science, 1998, 282 (5386), pp. 103 - 107.

[7] Farci, P., Strazzera, R., Alter, H. J., Farci, S., Degioannis, D., Coiana, A., Peddis, G., Usai, F., Serra, G., Chessa, L., Diaz, 
G., Balestrieri, A., Purcell, R. H. Early changes in hepatitis C viral quasispecies during interferon therapy predict the therapeutic outcome. Proceedings of the National Academy of Sciences of the United States of America, 2002, 99, pp. $3081-3086$.

[8] Kalinina, O., Norder, H., Mukomolov, S., Magnius, L. O. A natural intergenotypic recombinant of hepatitis $\mathrm{C}$ virus identified in St. Petersburg. Journal of Virology, 2002, 76 (8), pp. $4034-4043$.

[9] Raghwani, J., Thomas, X. V., Koekkoek, S. M., Schinkel, J., Molenkamp, R., van de Laar, T. J., Takebe, Y., Tanaka, Y., Mizokami, M., Rambaut, A., Pybus, O. G. Origin and Evolution of the Unique hepatitis $\mathrm{C}$ virus circulating recombinant form $2 \mathrm{k} / 1 \mathrm{~b}$. Journal of Virology, 2012, 86 (4), pp. $2212-2220$. 